\title{
Comet Halley Observed During the ESA Giotto Fly-By
}

\author{
H. U. Keller
}

Max-Planck-Institut für Aeronomie, Lindau, Germany

The major observational results of the detection of the nucleus of Comet Halley will be summarized with some emphasis on the images. A comparison to or context with ground based observations will be discussed. The paradigm set by the first observations of a cometary nucleus should be critically reviewed. The results of the VEGA missions and the recent observations of the nucleus of Comet Borrelly by DS-1 will also be considered. 